\title{
Role of enolase-1 in response to hypoxia in breast cancer: Exploring the mechanisms of action
}

\author{
JIE GAO $^{1^{*}}$, RONGRONG ZHAO ${ }^{1^{*}}$, YAN XUE $^{2^{*}}$, ZUOXING NIU $^{1}$, \\ KAI CUI ${ }^{3}$, FACHANG YU ${ }^{4}$, BO ZHANG ${ }^{1}$ and SHENG LI ${ }^{1}$ \\ ${ }^{1}$ Department of Hepatobiliary Surgery, Shandong Cancer Hospital, Jinan 276000; \\ ${ }^{2}$ Jinan Child Hospital, Jinan 250011; ${ }^{3}$ Key Laboratory for Rare and Uncommon Diseases of Shandong, \\ Jinan 250062; ${ }^{4}$ Shandong Academy of Medical Sciences, Jinan 250014, P.R. China
}

Received November 8, 2012; Accepted December 6, 2012

DOI: $10.3892 / o r .2013 .2269$

\begin{abstract}
In the present study, we investigated the effect of reduced enolase-1 expression in human umbilical vein endothelial cells (HUVECs)/MDA-MB-231 cells on the response to hypoxia and the possible mechanisms involved. Breast cancer cells transfected with enolase-1 siRNA were injected into mice to establish a tumor-bearing mouse model, and the correlation between enolase-1 expression and breast cancer angiogenesis, as well as its effect on the efficacy of radiation therapy were assessed. HUVECs were cultured in vitro, and transfected with enolase-1 siRNA. Following stable passage, $1.0 \% \mathrm{O}_{2}$ was used to induce hypoxia. The growth, proliferation, division and angiogenesis of HUVECs were observed using MTT assay, flow cytometry (FCM) and time-lapse video microscopy. The key regulatory molecules were detected using western blot analysis, two-dimensional (2-D) electrophoresis and matrix-assisted laser desorption/ionization time-of-flight mass spectrometry (MALDI-TOF-MS). The breast cancer cell line, MDA-MB-231, was cultured in vitro, and transfected with enolase-1 siRNA. The cells were injected into nude mice, and radiation therapy was administered. Tumor growth, angiogenesis in tumor tissues and apoptosis were observed, and the expression of the endogenous hypoxia marker, hypoxia inducible factor-1 $\alpha$ (HIF-1 $\alpha$ ), was detected using immunohistochemistry after the mice were sacrificed. A significant reduction in the hypoxia-induced apoptosis of HUVECs was observed in the control group compared with the endothelial cells transfected with enolase-1 siRNA. After the enolase-1 transfected breast cancer cells were injected into nude mice,
\end{abstract}

Correspondence to: Dr Sheng Li, Department of Hepatobiliary Surgery, Shandong Cancer Hospital, Jiyan Road 440, Jinan 276000, P.R. China

E-mail: drlisheng@sohu.com

*Contributed equally

Key words: endothelial cell, hypoxia, enolase-1, radiation therapy, proteomics tumor growth significantly declined, and the tumor volume and weight were reduced. Following treatment with radiation therapy, tumor size significantly decreased in both groups, and the highest reduction was observed in the transfected group. The reduction in enolase-1 expression significantly decreases the response to hypoxia and enhances the sensitivity of the cells to radiation therapy; therefore, enolase-1 may be a drug target for the treatment of breast cancer.

\section{Introduction}

Breast cancer is one of the most common malignant tumors in females, and hypoxia is observed during the development of the disease. Hypoxia is closely associated with malignant tumor progression, angiogenesis, invasion and the metastasis of tumor cells, as well as with the tolerance to treatment (1). In response to hypoxia, tumors form multiple vascular systems (angiogenesis) and improve the glycolytic rate. Glycolysis is a compensatory process of energy metabolism during hypoxia. As in most of the physiological activities in the body, adenosine triphosphate (ATP) is involved in the growth and proliferation of tumor cells and endothelial cells. In the context of hypoxia, compensatory adaptation is mainly achieved through the glycolytic process to obtain the necessary energy for life activities. The activation of the glycolysis-related metabolic enzyme enolase-1-mediated glycolytic pathway, improves the energy imbalance of hypoxic cells, promotes the transcription, proliferation and inhibits the apoptosis of the cellular oncogene, leading to the promotion of angiogenesis and affecting the response to hypoxia in tumor cells.

Enolase-1 is an oxidative stress protein derived from endothelial cells, which plays an important role in the conversion of 2-phosphoenolpyruvic acid in the glycolytic pathway. The overexpression of enolase-1 and other enzymes in the glycolytic process during hypoxia leads to the adaption of tumor cells to the energy requirement, and consequently increases survival and proliferation $(2,3)$, as well as the invasive and metastatic ability of the cells $(4,5)$. Yoo and Regnier (6) revealed that enolase-1, along with heat shock protein $\mathrm{A}$ and $\mathrm{C}$ were among the major oxidatively modified proteins associated with oxidative stress, playing important roles in response to hypoxia. It has been demonstrated that the glycolysis of endothelial cells 
increases during hypoxia, which is closely associated with angiogenic factors, such as tissue inhibitors of metalloproteinases (TIMPs), and cytokines such as tumor necrosis factor- $\alpha$ (TNF- $\alpha$ ) and interleukin- $\beta$ (IL- $\beta$ ). Differential RT-PCR has indicated that the expression of enolase-1 and TIMPs in human microvascular endothelial cells increases during hypoxia (7); enolase-1 and TIMPs promoted angiogenesis together, and a positive correlation was observed between them. Scharte et al (8) observed phenotypic changes and increased glycolysis in rat intestine-derived epithelial cells incubated in inflammatory medium [interferon- $\gamma(\mathrm{IFN}-\gamma), \mathrm{TNF}-\alpha$ and IL- $\beta$ )] under normoxic conditions, and increased hypoxia inducible factor-1 (HIF-1) DNA binding and enolase-1 expression. By highthroughput proteomic analysis, Somiari et al (9) demonstrated that the expression of enolase in human breast infiltrating ductal carcinoma tissues was higher than that in normal tissue. These findings suggest that enolase may promote the angiogenesis of tumor cells in response to hypoxia.

The expression of enolase-1 during hypoxia may be related to the JNK signaling pathway. Choi et al (10) found that vitexin inhibited the migration of rat pheochromocytoma (PC12) cells as well as their invasion rates, and that it also inhibited tube formation by human umbilical vein endothelial cells (HUVECs), acting in part, via the JNK pathway. In response to hypoxia in tumor cells, the correlation between hypoxia and enolase-1 and angiogenic factors, the role of the possible signaling pathway, and its correlation with oncogenes such as $c-m y c, p 53, B c l-2$ and Bax should be further investigated.

There is a subtle correlation between enolase- 1 and apoptotic factors. España et al (2) investigated the interaction of proteins in breast cancer cells transfected with $\mathrm{Bcl}-\mathrm{x}(\mathrm{L})$, and found that the metastatic activity of the cells was associated with the regulation of glycometabolism and amino acid metabolism. In addition, the expression of both enolase and aminoacylase-1 significantly increased in the cytoplasm, and a higher correlation was observed between them. It was speculated that the transfection and expression of $\mathrm{Bcl}-\mathrm{x}(\mathrm{L})$ coupled oxidative phosphorylation with glycolysis to protect against apoptosis. The findings further demonstrated that Bcl family, along with glycolytic metabolism, inhibited the anoikis of cancer cells, and enabled the interaction between the survived cells, which is in agreement with the study by Kabbage et al (28). They found that the expression of enolase- 1 and antioxidant protein (Mn-SOD) was significantly higher in breast cancer tissues compared to normal tissues. HIF-1 exhibits an anti-apoptotic role, and the mechanism involved may be associated with the activation of the target gene, vascular endothelial growth factor (VEGF). Yu et al (11) employed RNA interference (RNAi) to effectively and specifically inhibit the expression of HIF-1 in HUVECs. Following exposure to anoxia and reoxygenation, it was observed that the percentage of apoptotic HUVECs treated with RNAi was significantly greater than that of HUVECs not treated with RNAi. In addition, HIF is considered as a proapoptotic factor which downregulates $\mathrm{Bcl}-2$ expression, and hypoxia alters the microenvironment by inducing acidosis to increase the p53 level and promote apoptosis (12). Enolase-1 is activated during hypoxia and by HIF-1; therefore, the correlation between enolase-1 and apoptosis and HIF-1 seems justified.

Use of specific RNAi to inhibit the expression of enolase-1 in endothelial cells, may facilitate the understanding of its role in promoting the proliferation of vascular endothelial cells, inhibiting apoptosis, as well as promoting angiogenesis and the active transformation of the upstream and downstream molecules in the signaling pathway. The blockade of the pathway involved in the adaption to hypoxia in endothelial cells, promotes the hypoxic apoptosis of endothelial cells and inhibits angiogenesis, resulting in the inhibition of tumor growth, invasion and metastasis; this may provide references for increasing the sensitivity of radiotherapy and improving efficacy.

Previous studies have demonstrated that the tumor conditioned medium from the breast carcinoma cell line, MDA-MB-231, cells led to the upregulated expression of enolase-1 in HUVECs, the elevated reproductive and antiapoptotic ability of endothelial cells, increase in cell cycle progression and improved in vitro angiogenesis $(13,14)$. A number of previous studies have proven the critical role of enolase-1 in mediating transcription and proliferation (7). Based on previous studies, in the present study, endothelial and breast cancer cells were co-cultured and transfected with enolase-1 siRNA. HUVECs transfected or not with enolase-1 siRNA were exposed to hypoxia. The critical proteins in the enolase-1-mediated active proliferation of endothelial cells and the active transformation of angiogenesis of breast cancer cells responding to hypoxia were identified using cellular and molecular biological and proteomics techniques. In addition, enolase-1-transfected MDA-MB-231 cells were injected into nude mice and radiotherapy was administered to observe the effect of enolase-1 expression on the efficacy of radiotherapy.

\section{Materials and methods}

Cell culture and preparation of conditioned medium. The culture of primary HUVECs was carried out as previously described (14) with some modifications. Umbilical cords were collected from newborn healthy infants (informed consent was obtained and the study was approved by the ethics review committee of our hospital). The remaining blood in the umbilical vein was cleaned using sterile cold phosphate-buffered saline (PBS) at $4^{\circ} \mathrm{C}$ in the laminar flow hood and liquid leakage was checked. The umbilical vein was filled with $0.1 \%$ collagenase, and then incubated in a water bath at $37^{\circ} \mathrm{C}$ for $20 \mathrm{~min}$. The digestive fluid was then transferred into 50-ml centrifugal tubes, and centrifuged at 1,500 rpm for $15 \mathrm{~min}$. The supernatant was removed, and the complete M199 medium containing $100 \mu \mathrm{g} / \mathrm{ml}$ penicillin-streptomycin, $25 \mu \mathrm{g} / \mathrm{ml}$ endothelial cell growth factor, $1 \%$ glutamine and $20 \%$ fetal bovine serum was added into the tubes and the cells were cultured in Petri dishes. The HUVECs used in the present study were from the second or third passage, and at 3-4 days in the log phase cell growth was determined using the growth curve method. Under an inverted phase contrast microscope, immunohistochemistry for factor VIII-related antigen in the endothelial cells showed that the confluent monolayers of endothelial cells appeared flagstone-like. The cell viability was $>95 \%$, which was calculated using the trypan blue exclusion test, and the cell growth curve was plotted. The endothelial cells were synchronized by serum starvation and contact inhibition.

The MDA-MB-231 cell lines were cultured in M199 medium containing $10 \%$ fetal bovine serum, and the tumor cells were 
synchronized by serum starvation (cultured without serum for $24 \mathrm{~h}$ ). When the tumor cell growth reached $85 \%$ confluence, the cells were washed with PBS twice, and fresh M199 medium without serum was added $(4 \mathrm{ml})$, followed by culture at $37^{\circ} \mathrm{C}$ in an environment containing $5 \% \mathrm{CO}_{2}$ for $24 \mathrm{~h}$. The supernatant was collected, centrifuged at $100 \mathrm{x} \mathrm{g}$ to remove the cell debris, and was then sifted from the filtration membrane with a mesh size of $0.22 \mu \mathrm{m}$ and stored at $-20^{\circ} \mathrm{C}$ for the subsequent experiment as previously described (16).

Determination of cell proliferation. Cells were assigned to 4 groups according to the treatment protocols: i) c-nor group, HUVECs cultured under normoxic conditions; ii) s-nor group, HUVECs transfected with enolase-1 siRNA, and cultured under normoxic conditions; iii) $\mathrm{c}-1.0 \% \mathrm{O}_{2}$ group, HUVECs cultured in $1.0 \% \mathrm{O}_{2}$ (hypoxic conditions); iv) $\mathrm{s}-1.0 \% \mathrm{O}_{2}$ group, HUVECs transfected with enolase-1 siRNA, cultured in $1.0 \% \mathrm{O}_{2}$ (hypoxic conditions). The confluent monolayers of HUVECs were digested with pancreatin $(0.25 \%$ pancreatin $+0.1 \%$ EDTA), and then seeded onto 96 -well plates at a concentration of $5 \times 10^{3}$ cells per well. After $24 \mathrm{~h}$, the supernatant was removed and $200 \mu \mathrm{l}$ of various conditioned media were added and the different conditioned media were diluted with endothelial cell culture fluid at a ratio of 1:1 for $60 \mathrm{~h}$. After the supernatant was removed, the proliferation of HUVECs was detected using 3-(4,5)-dimethylthiahiazo(-z-y1)-3,5diphenytetrazoliumromide (MTT) assay.

Determination of cell cycle using flow cytometry (FCM). The HUVECs in the c-nor, s-nor, c- $1.0 \% \mathrm{O}_{2}$ and $\mathrm{s}-1.0 \% \mathrm{O}_{2}$ groups were digested with pancreatin $(0.25 \%$ pancreatin $+0.1 \%$ EDTA), and then seeded into culture flasks at a concentration of $5 \times 10^{3}$ cells per flask. After culturing at $37^{\circ} \mathrm{C}$ in $5 \% \mathrm{CO}_{2}$ overnight, $200 \mu \mathrm{l}$ of various conditioned media were added to the cells or the cells were treated with different protocols for $60 \mathrm{~h}$. Cells were then digested with pancreatin, harvested and centrifuged at 1,500 rpm for $5 \mathrm{~min}$, washed with PBS, fixed in $70 \%$ ethanol, and stained with propidium iodide. The cell cycle was detected using FCM.

Observation of morphology of angiogenesis. The culture flasks containing endothelial cells with $50 \%$ of confluence were placed on the loading platform of a thermostatic inverted biological microscope (Nikon, Tokyo, Japan), and the temperature at the work region was sustained at $37^{\circ} \mathrm{C}$. The mitosis of endothelial cells was transmitted to the time-lapse recorder (NV8085) via a low-light camera. The cassette speed of the camera was set at $72 \mathrm{~h}$. A fixed field of vision was selected and continuous recording was performed, and the video was played back at a normal speed to observe the dynamic process of the mitosis of endothelial cells. The images were sampled using the microcirculation digital imaging processor.

A total of $250 \mu \mathrm{l}$ Matrigel matrix ( $20 \mathrm{~g} / \mathrm{l})$ was sampled using a pre-cooled sterile pipet, and transferred to pre-cooled 24-well plates, which were then placed at $37^{\circ} \mathrm{C}$ in a box containing $5 \%$ $\mathrm{CO}_{2}$ to congeal the Matrigel matrix. The endothelial cells were seeded onto the gels at a concentration of $5 \times 10^{4}$ cells per well, and cultured at $37^{\circ} \mathrm{C}$ in an incubator containing $5 \% \mathrm{CO}_{2}$ for $24 \mathrm{~h}$. The formation of tube-like structures was recorded using time-lapse video microscopy.
Detection of apoptosis. The endothelial cells, which were synchronized by contact inhibition, were seeded at a concentration of $5 \times 10^{4}$ cells per flask onto culture flasks coated with gelatin. After $24 \mathrm{~h}$ when the cells were fully adhesive to the flask walls, the cells were exposed to normoxic and hypoxic conditions for $24 \mathrm{~h}$. The detection of apoptosis was carried out following the manufacturer's instructions (Annexin V-FITC, Takara Bio). Cells were washed in PBS, digested with pancreatin, and centrifugated at 1,000 rpm 3 times. The sediment was suspended using buffer solution, and supplemented with $10 \mu \mathrm{l}$ of Annexin V-FITC (20 mg/l) and $5 \mu \mathrm{l}$ of propidium iodide (50 mg/l), and finally, $300 \mu \mathrm{l}$ of buffer solution were added. Apoptosis was detected using FCM.

In addition, apoptosis was determined using the terminal deoxynucleotidyl transferase-mediated dUTP-biotin nick end-labeling (TUNEL) apoptosis assay kit (Roche), and TdT was replaced by labeling solutions as the positive controls. The nucleus stained in pale brown or brown was considered positive (apoptotic cells). Each section was observed for at least 3 continuous fields of vision at high magnification, and the number of apoptotic cells in 1,000 cells in each field was recorded.

Two-dimensional (2-D) electrophoresis. The endothelial cells cultured under various conditions for $24 \mathrm{~h}$ were harvested, washed in cold PBS 4 times, and cell lysis solution was added for $30 \mathrm{~min}$, followed by centrifugation at $12,000 \mathrm{rpm}$ in $4^{\circ} \mathrm{C}$ for $15 \mathrm{~min}$. The supernatant was collected, which was the total protein component. The protein concentration was determined using the Bradford method, and then stored at $-80^{\circ} \mathrm{C}$ for the subsequent experiment.

1-D electrophoresis was performed using Bergman's method on an IPGphor isoelectric focusing system. At a pH of 3-10, 2 dry strips of $18 \mathrm{~cm}$ in length were placed in a trough of a strip swelling tray after removal of the protective membrane. Protein sample of $1 \mathrm{mg}$ was added, and the rehydration of the IPG strips was performed. Isoelectric focusing was performed automatically, and the voltage and current displayed $3316 \mathrm{~V}$ and $50 \mu \mathrm{A}$ at the end of isoelectric focusing. After electrophoresis, 2 IPG strips were placed in balanced solution (D1/D2), and then vibrated for $15 \mathrm{~min}$ in a shaker. According to the volume of the IPG conversion kit, $12 \%$ gels at a size of $200 \mathrm{~mm} \mathrm{x}$ $200 \mathrm{~mm}$ x $1 \mathrm{~mm}$ were prepared. The balanced IPG strips were transferred onto the gels, and blocked using $0.5 \%$ agarose. At $20^{\circ} \mathrm{C}$, electrophoresis at a constant current of $15 \mathrm{~mA}$ for each strip was performed until the bromophenol blue reached the lower edge of the glass plate.

Following 2-D electrophoresis, gels were placed in stationary liquid (50\% anhydrous ethanol and $10 \%$ ethanoic acid) for $3 \mathrm{~h}$, washed with deionized water, stained with Coomassie brilliant blue R250, vibrated for $1 \mathrm{~min}$ on a shaker, decolorized twice, for $30 \mathrm{~min}$ each time, and finally washed with distilled water. Images were obtained by using an imaging scanner. The 2-D gels were scanned and inputted into a computer, and the 2-D gel profile was analyzed using PDQuest software (Bio-Rad). The profile was compared 3 times, and the standard profile was obtained. The standard profiles of tissues treated with different media were matched, and variations in protein expression caused by hypoxia were analyzed. 
The differentially expressed protein spots were cut from the gels, and digested using Bergman's method. The proteins were identified using the matrix-assisted laser desorption/ionization time-of-flight mass spectrometry (MALDI-TOF-MS) on a mass spectrometer (Voyager-DE PRO). The peptide mass fingerprint maps, molecular weight and isoelectric point of the differentially expressed protein spots were input into a computer, and the matched proteins were searched in the Swiss-Prot/TrEMBL database using PeptIdent searching software, and the functions of the proteins were inquired.

Western blot analysis. The endothelial cells cultured under various conditions for $24 \mathrm{~h}$ were harvested, washed in cold PBS 4 times, and cell lysis solution was added, followed by centrifugation at $12,000 \mathrm{rpm}$ in $4^{\circ} \mathrm{C}$ for $15 \mathrm{~min}$. The supernatant was collected, which was the total protein component. The protein concentration was determined by using Bradford method, and then stored at $-80^{\circ} \mathrm{C}$ for the subsequent experiment.

Total protein solution (30 $\mu \mathrm{g})$ was sampled for SDS-PAGE. The protein was transferred onto a PVDF membrane (40 min) at a suction power of $70 \mathrm{~mA} / \mathrm{cm}^{2}$ using the semi-dry transfer method. The membrane was blocked with $5 \%$ non-fat milk powder at room temperature for $1 \mathrm{~h}$, and anti-cyclin D1/E (primary antibody) was then added at $4^{\circ} \mathrm{C}$ overnight, followed by washing in TBST 3 times, for 15 min each time. The membrane was then incubated in HRP-labeling second antibody diluted at a ratio of 1:1,000 at room temperature for $1 \mathrm{~h}$, washed in TBST 3 times, for 15 min each time, and finally stained using the ECL + western blotting detection system.

Establishment of tumor nude mouse model and radiotherapy. The well cultured MDA-MB-231 cells in the exponential phase were digested with pancreatin, and then $100 \mu 1$ of cell suspension (containing about $2 \times 10^{6}$ cells) were subcutaneously injected into the left armpit of 3-4-week-old female BALB/c nude mice. The mice were raised in a sterile environment, and the general condition of nude mice and tumor growth were observed and recorded each day. At approximately 1 month after the injection, the tumor size had reached approximately $2 \mathrm{~mm}^{3}$. Nude mice were randomly assigned to 5 groups (10 animals in each group). Group 1 (c-nor), mice were injected with MDA-MB-231 cells and received no treatment; group 2 (s-nor), mice were injected with enolase-1 siRNA-transfected MDA-MB-231 cells and received no treatment; group $3(c+r)$, mice were injected with MDA-MB-231 cells and received radiotherapy twice a week; group $4(s+r)$, mice were injected with enolase-1 siRNA-transfected MDA-MB-231 cells and received radiotherapy twice a week; and group 5, served as the controls. The physical state of the nude mice was monitored each day, and body weight was measured twice a week. The tumor size was measured using a vernier caliper twice a week. At 4 weeks after treatment, the animals were sacrificed. The entire tumor was collected and weighed. Tumor inhibition was calculated using the following formula: inhibition rate of tumor $(\%)=$ (tumor weight in the control group - tumor weight in the treatment group)/tumor weight in the control group $\mathrm{x} 100 \%$.

The fresh tumor tissues were fixed in formaldehyde, embedded with paraffin wax, and cut into sections. The sections were dewaxed with xylene, and placed in $3 \% \mathrm{H}_{2} \mathrm{O}_{2}$ ethanol for $10 \mathrm{~min}$ to remove the activity of endogenous peroxidase. Microwave antigen retrieval was performed at $92-98^{\circ} \mathrm{C}$ with a power of $600 \mathrm{~W}$ for $10 \mathrm{~min}$, the tissues were then blocked with 5-10\% normal goat serum (diluted with PBS) for $10 \mathrm{~min}$, and secondary antibody was added in the EnVision ${ }^{\mathrm{TM}}$ system at $37^{\circ} \mathrm{C}$ for $10 \mathrm{~min}$.

Sections were observed carefully under an optical microscope at a low magnification (x10) to identify the hot spot in the tumor microvessel. The pale brown capillary lumen which was positive for CD31 staining and significantly distinct from the tumor cells and connective tissues was calculated as a vessel. According to the capillary lumen size and capillary wall thickness, the artery and vein were excluded. The diameter of the selected capillary lumen was $\leq 20 \mu \mathrm{m}$. The microvessel was calculated by 3 fields of vision in each section, and the mean value was described as the microvessel density (MVD). The area density and number density of the microvessel in the selected sites were estimated using the CMIAS multi-function real color pathological imaging analysis system. Area density, area of microvessel $\left(\mathrm{mm}^{2}\right)$ /area of section $\left(\mathrm{mm}^{2}\right)$; number density, number of microvessel/area of section $\left(\mathrm{mm}^{2}\right)$.

Immunohistochemical staining to detect hypoxic tumor microenvironment. At $1 \mathrm{~h}$ after sacrifice, the nude mice were peritoneally injected with $60 \mathrm{mg} / \mathrm{kg}$ hypoxyprobe. The principle of hypoxyprobe used for the detection of hypoxia is shown in Fig. 1. If the oxygen concentration is $<14 \mu \mathrm{M}$ in tissues, the intracellular electron is transferred to the hypoxyprobe via the electric transfer chain, producing an amino negative ion. After 4 ions are transferred to the hypoxyprobe, hydroxylamine derivatives are formed, which interact with the peptides and proteins containing sulfhydryl groups in hypoxic cells, resulting in the formation of stable adducts which can be detected using immunohistochemistry. The hypoxic tumor microenvironment was examined following the manufacturer's instructions (Hypoxyprobe ${ }^{\mathrm{TM}}-1$ Plus kit, Seebio Biotech, Inc.).

Statistical analysis. All experiments were repeated at least 3 times. All data were expressed as the means \pm standard deviation (SD), and all statistical analyses were performed using the statistical software SPSS version 10.0. The t-test was used to compare differences between groups. A P-value $<0.05$ was considered to indicate a statistically significant difference.

\section{Results}

Morphology and growth of confluent HUVEC monolayers. Under an inverted phase contrast microscope, we observed flagstone-like confluent monolayers of cells (Fig. 2), which were identified as endothelial cells using immunohistochemical staining for factor VIII-related antigen (Fig. 3). During the passage of HUVECs, the growth rate of cells at the third passage was slightly slower than that of the cells at the second passage, and the cell growth proceeded to the log phase after culturing for 3-4 days.

Under an inverted phase contrast microscope, it was observed that the endothelial cells grew vigorously under normoxic conditions, with many split phase cells. In the prophase of mitosis, cells shrunk, cell adhension to the culture wall was reduced, and condensed chromatin aggregates were 


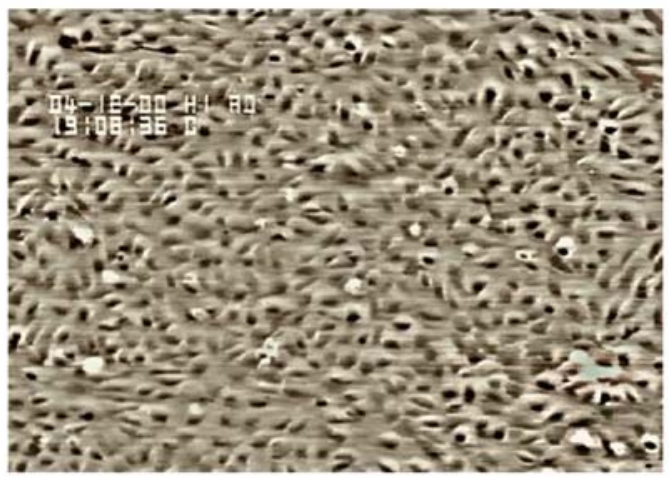

Figure 1. Endothelial cell morphology. The cells show a typical pebble-like shape under a microscope at a low magnification (x100).

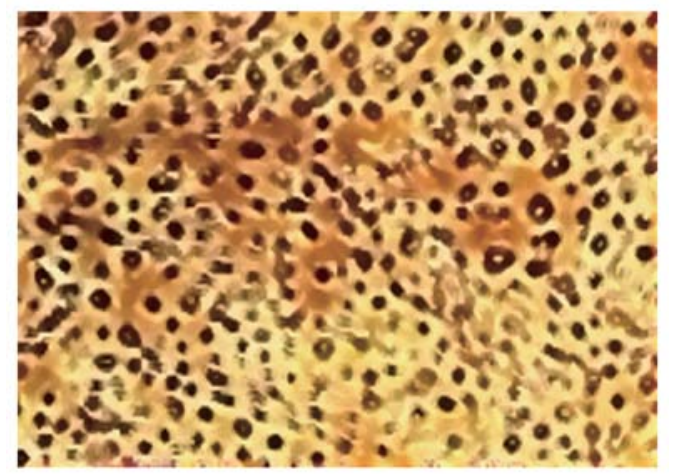

Figure 2. Immunohistochemical identification of Factor VIII. The brownstained dots are positive cells, and the positive rate was $95 \%$.

observed. Refraction halos were observed around the cells under an optical microscope (Fig. 4). There were also dumbbell-like cells observed in the anaphase. Following transfection with enolase-1 siRNA under normoxic conditions, the growth rate of the endothelial cells was slightly lower than that of the controls, with a slight reduction in split phase cells and a slightly slower rate of cytokinesis. Time-lapse video microscopy showed that the cytoplasms of the daughter cells present in the telophase formed extensions at a faster rate in the control group (5-6 min) compared to the enolase-1 siRNA-transfected cells (12-13 $\mathrm{min})$.

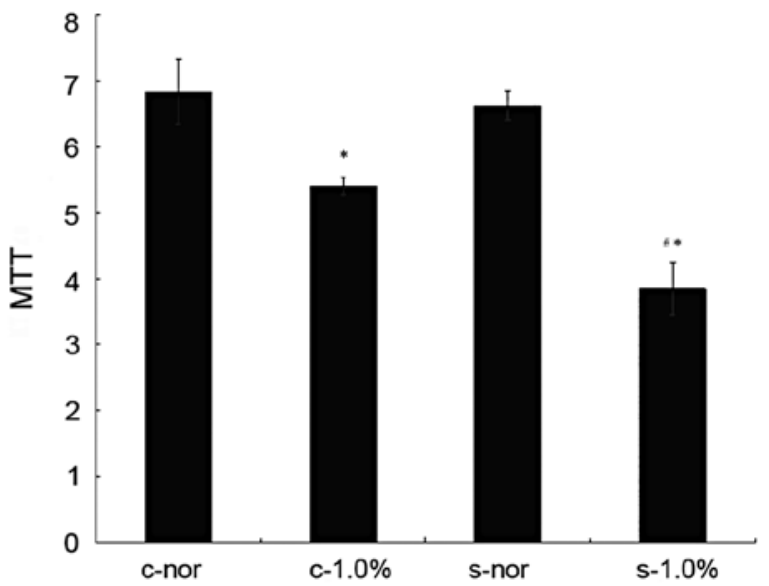

Figure 4. Effect of transfection with enolase-1 siRNA on cell proliferation. ${ }^{\#} \mathrm{P}<0.05$ vs. the $1.0 \% \mathrm{O}_{2}$ group; ${ }^{*} \mathrm{P}<0.05$ vs. the c-nor group.

Effect of hypoxia $\left(1.0 \% \mathrm{O}_{2}\right)$ on proliferation and viability of endothelial cells before and after transfection with enolase-1 SiRNA. Before and after the transfection of HUVECs, no significant difference in the proliferation and viability was observed under normoxic conditions. Under hypoxic conditions, the growth of the cells in both groups was reduced, and the proliferative ability significantly decreased in the cells transfected with enolase-1 siRNA (Fig. 1). Following transfection with enolase-1 siRNA, the cell growth was reduced compared with that before transfection, and the number of cells in the G1 phase increased while it was reduced in the $\mathrm{S}$ phase (Table I).

Effect of hypoxia on apoptosis of HUVECs before and after transfection with enolase-1 siRNA. Following transfection with enolase-1 siRNA, the number of apoptotic HUVECs slightly increased, and significantly increased following exposure to hypoxia, which was characterized by late apoptotic cells. The apoptotic rate of HUVECs increased from 5.8 to $10.9 \%$ in the c-nor group, whereas from 6.5 to $13.5 \%$ in the s-nor group (Fig. 5).

Effect of hypoxia on expression of apoptosis-related factors of HUVECs before and after transfection with enolase-1 siRNA. Western blot analysis revealed that transfection with
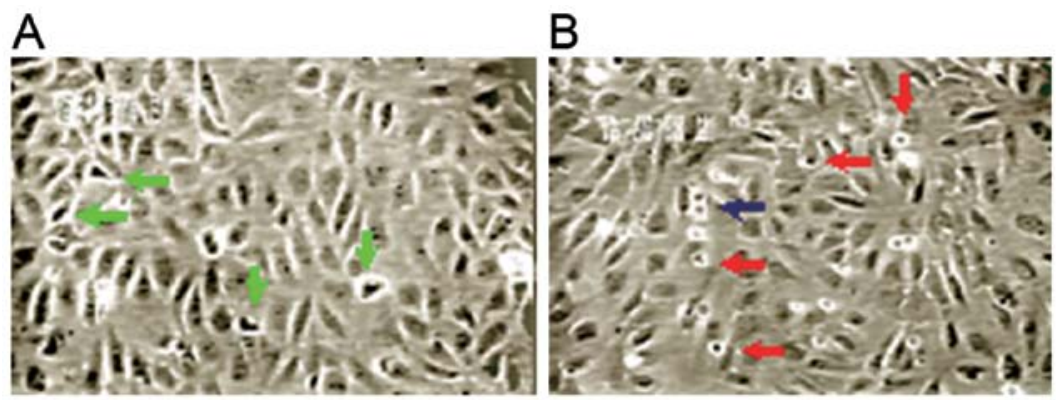

Figure 3. Endothelial cells cultured under normal oxygen conditions (A) before and (B) after enolase-1 transfection. (A) There are many cells with a distinctive glistening appearance during the prophase (shown by the green arrows). (B) There are many cells of a small volume during the metaphase, with a round shape, and the surrounding glistening rings are large (shown by the red arrows). Dumbbell-shaped cells (shown by the blue arrows) in the late and final stages of mitosis are present. 
A

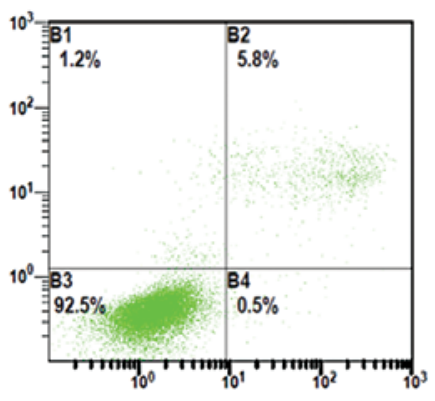

B

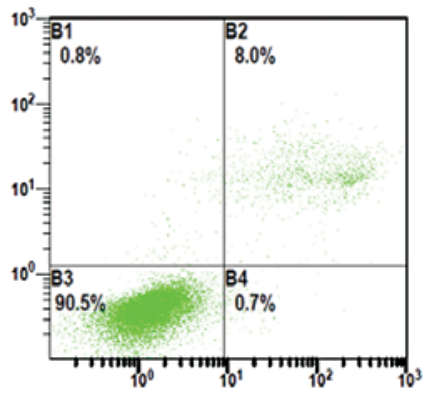

C

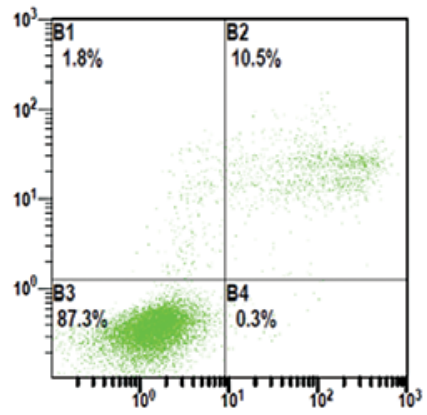

D

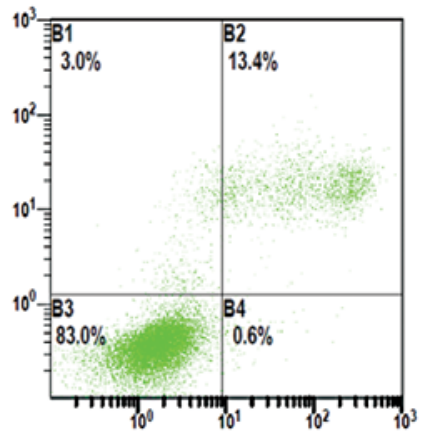

Figure 5. The number of apoptotic cells under normal oxygen and hypoxic conditions. (A) The c-nor group; (B) the s-nor group; (C) the c-1.0\% $\mathrm{O}_{2}$ group; (D) the $-1.0 \% \mathrm{O}_{2}$ group.

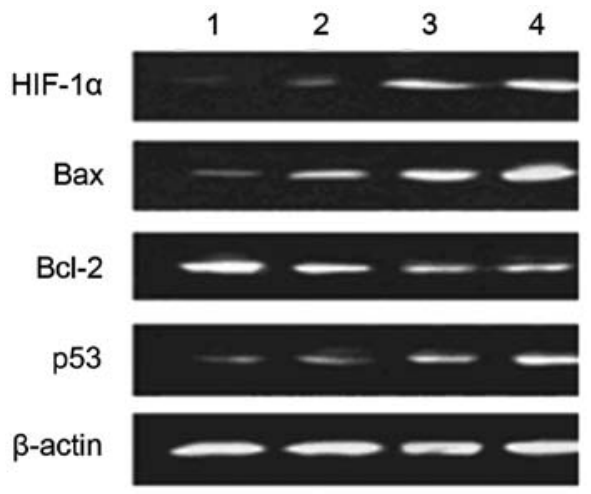

Figure 6. Western blot analysis to detect the effect of hypoxia on the expression of apoptosis-related factors in HUVECs prior to and after siRNA transfection. Lane 1: c-nor group, HUVECs cultured under normoxic conditions; lane 2: s-nor group, HUVECs transfected with enolase-1 siRNA and cultured under normoxic conditions; lane 3: $\mathrm{c}-1.0 \% \mathrm{O}_{2}$ group, HUVECs cultured in $1.0 \%$ $\mathrm{O}_{2}$ (hypoxic conditions); lane 4: s-1.0\% $\mathrm{O}_{2}$ group, HUVECs transfected with enolase-1 siRNA, cultured in $1.0 \% \mathrm{O}_{2}$ (hypoxic conditions).

enolase-1 siRNA almost had no effect on the expression of HIF- $1 \alpha$; HIF- $1 \alpha$ is not affected by its downstream product, enolase-1. No expression of HIF-1 $\alpha$ was observed under normoxic conditions; however, HIF-1 $\alpha$ expression significantly increased under hypoxic conditions. After transfection, the expression of Bax and p53 notably increased following exposure to hypoxia. It is indicated that enolase-1 may exert an effect on apoptosis by altering the expression of certain apoptotic factors (Fig. 6).

Proteomics analysis. The whole-cell proteins of HUVECs exposed to hypoxia for $10 \mathrm{~h}$ were separated by using 2-D electrophoresis, and following staining with Coomassie Brilliant Blue solution, the 2-D gel map was obtained. The experiments were repeated 3 times, and the protein dots were $518 \pm 31$ and $482 \pm 27$ on the maps from cells in the 2 groups, revealed by PDQuest 6.2 software. Compared with the non-transfected group, protein expression was significantly reduced in the transfected group $(\mathrm{P}<0.05)$. The isoelectric points of the proteins ranged from 5.0 to 9.0 , and the molecular weight was between 21 and $97 \mathrm{kDa}$. Matching analysis by PDQuest identified 8 differentially expressed proteins, including 5 downregulated and 3 upregulated.
Table I. HUVEC counts and proportions in different phases of the cell cycle in the $c$-nor and s-nor groups (mean $\pm S D, n=3$ ).

\begin{tabular}{lcccc}
\hline & & \multicolumn{3}{c}{$\begin{array}{c}\text { Proportion of cells in different } \\
\text { phases of cell cycle }(\%)\end{array}$} \\
\cline { 3 - 5 } Group & $\begin{array}{c}\text { Cell count } \\
\left(\mathrm{x} 10^{5}\right)\end{array}$ & G1 phase & S phase & $\begin{array}{c}\text { G2/M } \\
\text { phase }\end{array}$ \\
\hline c-nor & $10.69 \pm 0.55^{\mathrm{a}}$ & $68.33 \pm 2.14$ & $15.87 \pm 0.55$ & $15.83 \pm 2.04$ \\
s-nor & $9.65 \pm 0.16$ & $71.50 \pm 1.76$ & $13.76 \pm 1.31$ & $14.03 \pm 0.39$ \\
\hline
\end{tabular}

Table II. Search parameters of PeptIdent software.

\begin{tabular}{ll}
\hline Parameter & \multicolumn{1}{c}{ Parameter setting } \\
\hline Database searched & Swiss-Prot \\
Species searched & Homo sapiens (human) \\
Digestive enzyme used & Trypsin \\
Peptide mass accuracy & $\pm 1.5 \mathrm{Da}$ \\
Methionine & Oxidized \\
Cysteine & Carbamidomethylation \\
Peptide masses are & Monoisotopic \\
Number of uncleaved sites & $1-2$
\end{tabular}

The differentially expressed proteins were analyzed using MALDI-TOF-MS, and the obtained peptide mass fingerprinting $(\mathrm{PMF})$ profiles were matched to the standard PMF in the Swiss-Prot database. The PeptIdent software was used for inquery and identification (Table II). The matching results including the Swiss-Prot accession number, proteins identified, matching rate and sequence coverage are shown in Table III, and the searched protein mass map and the matched peptides are listed. Phospholipid transfer protein (PLTP), plays a role in promoting lipid transfer, antioxidant activity and maintaining normal functions in cells. HLP2, a DEAD-binding protein, promotes the transcription and extension of the DNA strand. C2 esterase promotes the adhension of leucocytes to 
Table III. Differentially expressed proteins identified using PeptIdent software.

\begin{tabular}{lcccl}
\hline Spot & $\begin{array}{c}\text { Swiss-Prot } \\
\text { (Accession no.) }\end{array}$ & $\begin{array}{c}\text { Matching } \\
\text { rate (\%) }\end{array}$ & $\begin{array}{c}\text { Sequence } \\
\text { coverage (\%) }\end{array}$ & \multicolumn{1}{c}{ Protein identified } \\
\hline Downregulated protein & P55059 & 38 & 15 & $\begin{array}{l}\text { Phospholipid transfer protein precursor } \\
\text { (PLTP precursor) } \\
\end{array}$ \\
& Q00571 & 53 & 21 & $\begin{array}{l}\text { DEAD-box protein 3 (HLP2) } \\
\text { C1 esterase }\end{array}$ \\
& P89871 & 36 & 12 & Pyruvate kinase \\
P14618 & 62 & 27 & ATP-dependent DNA helicase II \\
P13010 & 56 & 12 & 5'-TG-3' interacting factor (TGIF) \\
& Q15583 & 29 & 18 & FR- $\beta$ \\
& P14207 & 28 & 23 & Stomatin \\
\hline
\end{tabular}

A

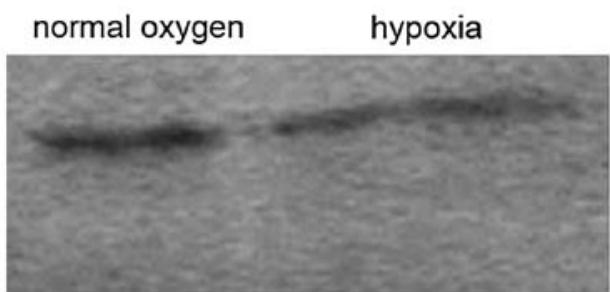

B normal oxygen hypoxia

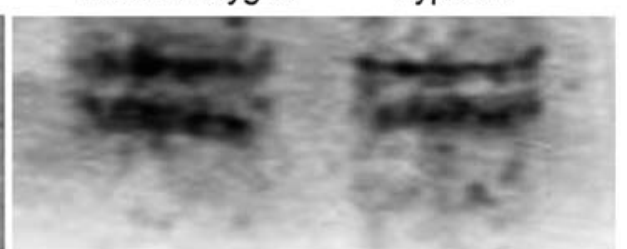

Figure 7. Western blot analysis to determine the expression of cyclin D1/E protein. (A) The total proteins from the endothelial cells under hypoxic conditions, particularly the moderate- and low-molecule proteins, was less than that under normoxic conditions. (B) The low expression of cyclin D1/E was observed, notably under normoxic conditions.

endothelial cells and regulates matrix metabolism. Pyruvate kinase, is a key enzyme for mediating glucose catabolism and provides energy for cell life activities. 5'-TG-3' interacting factor (TGIF), a co-inhibitor of Smad, inhibits DNA transcription induced by TGF- $\beta$. FR- $\beta$ mediates folic acid metabolism and negatively regulates cell proliferation. ATP-dependent DNA helicase (DNA-repairing protein), sustains and promotes normal cell cycle. Stomatin negatively mediates the membrane permeability of monovalent positive ions and is involved in assembly and organization of intracellular cytoskeletal protein actin.

SDS-PAGE and western blot analysis of the total proteins from the endothelial cells before and after exposure to hypoxia for $24 \mathrm{~h}$ showed that the number of proteins under hypoxic conditions, particularly the moderate- and low-molecule proteins, was less than that under normoxic conditions (Fig. 7). The low expression of cyclin D1/E was observed, notably under normoxic conditions. However, the polyclonal antibodies used in the current study led to cross-reactions or isomers.

Angiogenesis. In the 24-well plates coated with Matrigel matrix, following overnight culture, the endothelial cells formed typical tube-like structures, and no significant difference was observed between the transfected and non-transfected cells. However, large tube-like structures with low density were observed in the non-transfected cells, while many cord-like structures of a small volume were observed in the endothelial cells transfected with enolase-1 siRNA. Under hypoxic conditions, there were no obvious tube-like structures observed in the transfected and non-transfected cells (Fig. 8).

Tumor size. The largest tumor size was observed in the c-nor group, while significant reduced tumor sizes were detected in the s-nor and $s+r$ groups. Following radiotherapy, the tumor sizes were significantly reduced compared with the untreated groups and the highest reduction was observed in the $\mathrm{s}+\mathrm{r}$ group (Fig. 9).

$M V D$. After the tumor cells transfected with enolase-1 siRNA were injected into nude mice, the angiogenesis present in the tumor tissues is shown in Fig. 10. It was observed that less angiogenesis was detected in the nude mice receiving an injection with transfected cancer cells in comparison with the control group, which demonstrated the role of enolase-1 in angiogenesis.

The tumor tissue sections were stained using fluorescent TUNEL, and it was found that the number of apoptotic cells reduced in the control group. The number of apoptotic cells increased following radiotherapy. The number of apoptotic cells transfected with enolase-1 siRNA also increased, and this was further enchanced following radiotherapy (Fig. 11).

Low oxygen content and increased hypoxia were detected within the tissues of the non-transfected cells, and the hypoxic 

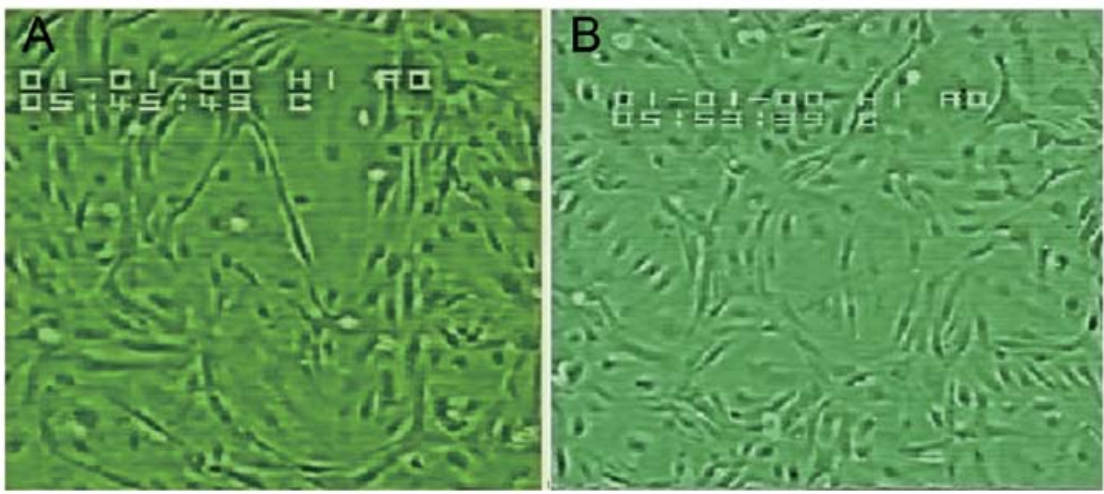

Figure 8. Variation in tube-like structure of HUVECs (A) before and (B) after transfection with enolase-1 siRNA. There is no significant difference in the total area of tube-like structures before and after transfection; however, differences in morphology and density are observed.

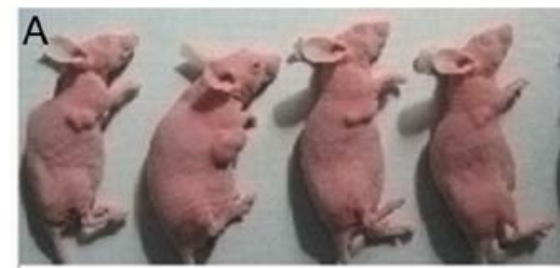

C

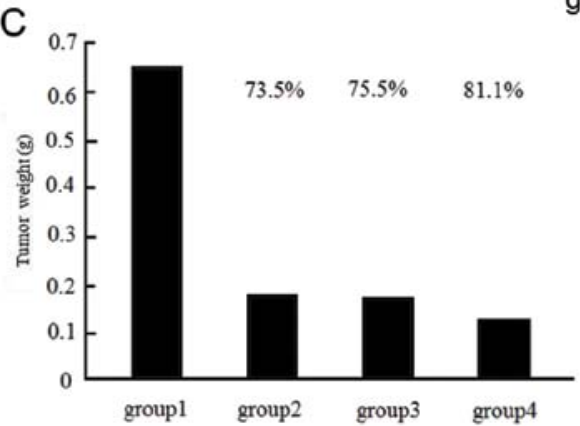

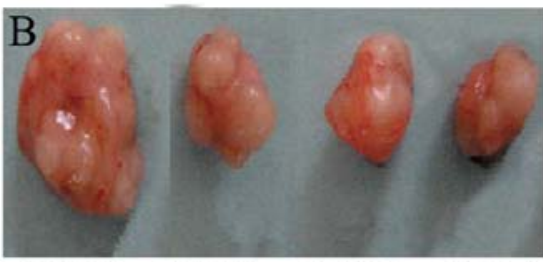

group 1 group 2 group 3 group 4

Figure 9. (A) Tumor size in the nude mice of different groups. (B) Tumor size in the different groups. The largest tumor size was observed in group 1 (c-nor), mice were injected with MDA-MB-231 cells and received no treatment; significant reduced tumor sizes were detected in group 2 (s-nor), mice were injected with enolase-1 siRNA-transfected MDA-MB-231 cells and received no treatment; group $3(\mathrm{c}+\mathrm{r})$, mice were injected with MDA-MB-231 cells and received radiotherapy twice a week; the highest reduction were detected in group $4(\mathrm{~s}+\mathrm{r})$, mice were injected with enolase-1 siRNA-transfected MDA-MB-231 cells and received radiotherapy twice a week. (C) Tumor weight in the different groups
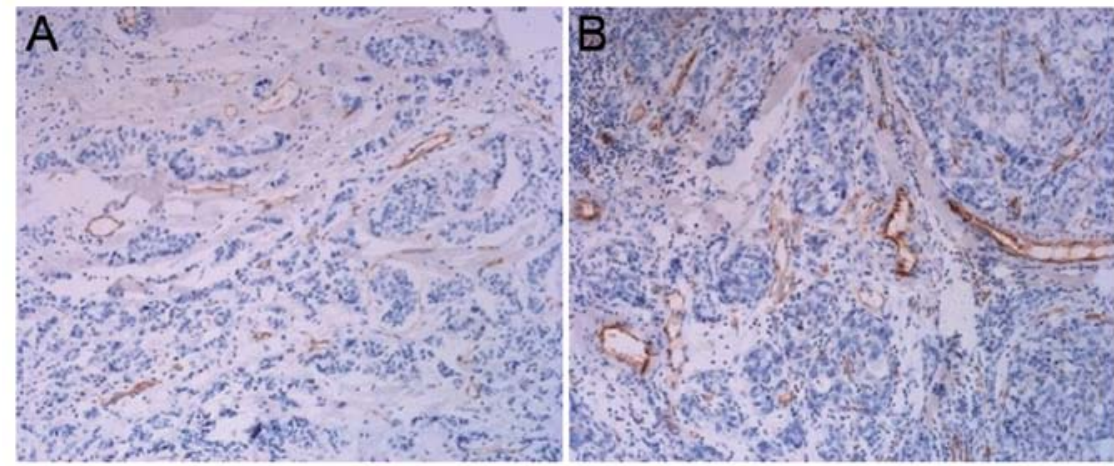

Figure 10. Angiogenesis in tumor tissues. (A) Tumor tissues transfected with enolase-1 siRNA; (B) control.

state was reduced but remained high following radiotherapy. Following transfection with enolase-1 siRNA, the hypoxic state was reduced, and further decreased following radio- therapy; however, there was no significant difference before and after radiotherapy. We hypothesized that a reduction in hypoxia is associated with a reduction in cell proliferation 
A
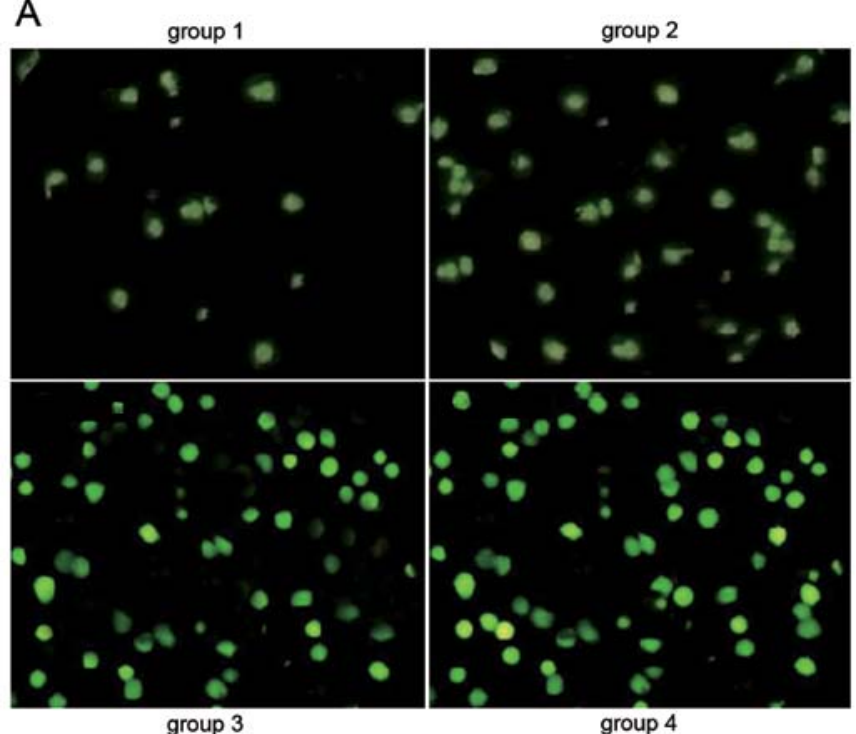

B

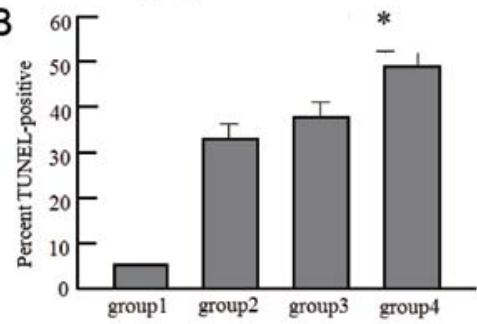

Figure 11. Apoptosis in tumor tissues detected by TUNEL. (A) The number of apoptotic cells reduced in group 1 (c-nor), mice were injected with MDA-MB231 cells and received no treatment; the number of apoptotic cells increased in group 2 (s-nor), mice were injected with enolase-1 siRNA-transfected MDA-MB-231 cells and received no treatment; the number of apoptotic cells increased in group $3(\mathrm{c}+\mathrm{r})$, mice were injected with MDA-MB-231 cells and received radiotherapy twice a week; the largest number of apoptotic cells increased in group $4(\mathrm{~s}+\mathrm{r})$, mice were injected with enolase-1 siRNAtransfected MDA-MB-231 cells and received radiotherapy twice a week. (B) The percent TUNEL-positive in the different groups.

and oxygen requirement following transfection with enolase-1 (Fig. 12).

\section{Discussion}

Glycolysis is a compensatory process of energy metabolism during hypoxia. The overexpression of a number of enzymes during the process of glycolysis leads to the adaption of tumor cells to the energy requirement, resulting in increased survival and proliferation $(2,3)$, as well as an increased invasive and metastatic ability $(4,5)$. For example, hexokinase, lactate dehydrogenase and glyceraldehyde-3-phosphate dehydrogenase present a regulatory function of transcription; glucose-6-phosphateisomerase has a function of mediating cell migration; glucokinase, hexokinase and glyceraldehyde-3-phosphate dehydrogenase mediate apoptosis, which demonstrates the correlation between metabolic enzymes and transcription mediation and cell proliferation. It is critical that the metabolic enzyme is targeted when investigating the pathological and physiological process of angiogenesis induced by hypoxia in breast cancer.

The correlation between the oncogene and adaptation to hypoxia may be connected by HIF-1 $\alpha$ and the metabolic
A
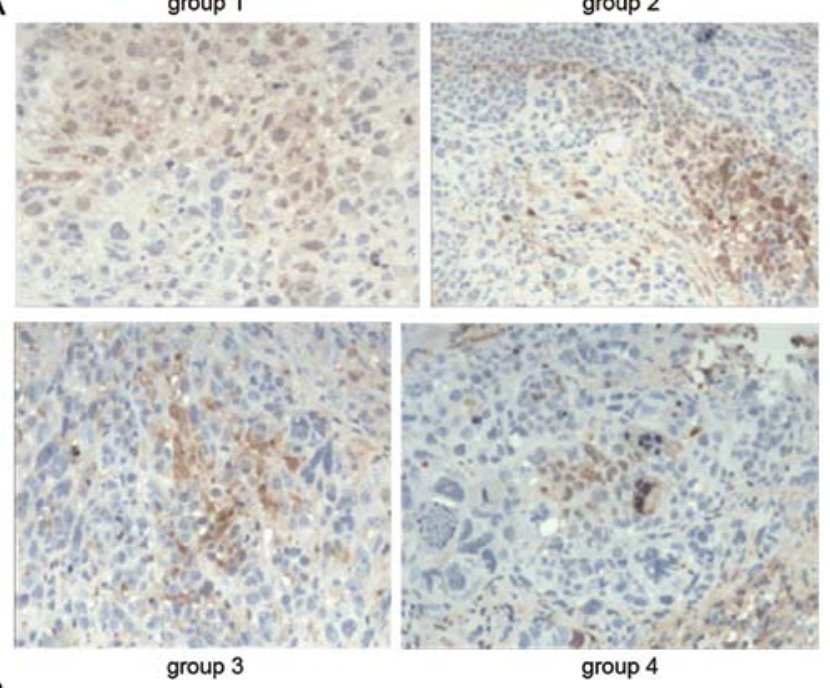

B

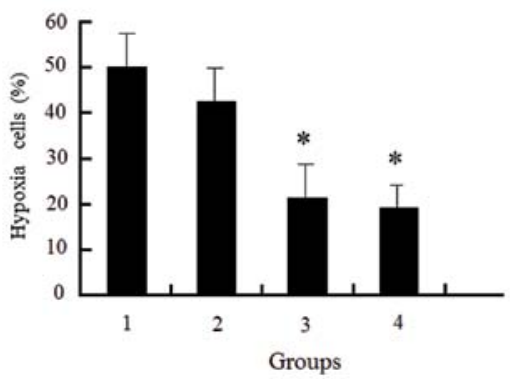

Figure 12. (A) Cell hypoxia after radiation therapy. Group 1 (c-nor), mice were injected with MDA-MB-231 cells and received no treatment; group 2 (s-nor), mice were injected with enolase-1 siRNA-transfected MDA-MB-231 cells and received no treatment; group $3(\mathrm{c}+\mathrm{r})$, mice were injected with MDA MB-231 cells and received radiotherapy twice a week; group $4(\mathrm{~s}+\mathrm{r})$, mice were injected with enolase-1 siRNA-transfected MDA-MB-231 cells and received radiotherapy twice a week. (B) Hypoxia cells (\%) in the different groups.

enzyme, enolase-1. The $v$-SRC oncogene increases the expression of $H I F-1, V E G F$ and enolase-1 under normoxic and hypoxic conditions, which is associated with the increased transcription of reporter genes containing cis-acting hypoxiaresponse elements from the VEGF and enolase-1 genes (17). The correlation between the metabolic sensor and transcription is connected directly or indirectly by many metabolic enzymes, which not only exert metabolic effects, but also regulate apoptosis and cell migration. The Myc promoter-binding protein MBP-1 is an inhibitor of Myc transcription, and shows $95 \%$ sequence homology to enolase-1. Currently, it is regarded as a product of the selective translation of enolase-1. In lung cancer, the downregulated expression of enolase-1 is related to poor prognosis. It is thought that Myc induces glycolysis through the negative feedback regulation of enolase-1 (18). In this study, MTT assay and cell cycle analysis revealed that under normoxic conditions, the growth and division of the endothelial cells transfected with enolase-1 siRNA were slightly weaker than those of the non-transfected cells; however, no significant difference was observed, which is consistent with the hypoxic induction of enolase-1. In addition, the results indicated that enolase-1 promoted cell proliferation, although the effect was not evident under normoxic conditions. 
The cells in the 2 groups responded differently to hypoxia; the number of hypoxic apoptotic cells in the control group was significantly lower than that in the transfected group, which further demonstrated the role of enolase-1 in adaptation to hypoxia in cells. The effect of enolase-1 on cell proliferation may be associated with the mediation of the expression of the oncogene and cell cycle-related protein. Western blot analysis revealed a variation in cyclin E1 expression, which was estimated to be associated with the cell cycle progression. A significant difference in the expression of Bax and p53 was observed under hypoxic conditions. Further studies should be carried out to investigate the signal transduction molecules. 2-D electrophoresis and MALDI-TOF-MS revealed 3 upregulated proteins and 7 downregulated proteins. In addition, proteomics analysis showed changes in the expression of 3 DNA transcription-related proteins in the siRNA-transfected endothelial cells. ATP-dependent DNA helicase, also termed DNA-repairing protein, maintains and promotes the normal cell cycle. The increased expression of HLP2, a member of the DEAD-binding protein family, promotes the transcription and extension of DNA extension (19).

The expression of TGIF $(20,21)$, a homeobox protein, was reduced. TGIF, as a co-inhibitor of the transcription factor Smad, inhibits the transcription induced by TGF- $\beta$ and steroid hormone. The mechanisms may be explained by the following 2 aspects: i) TGIF activates histone deacetylase until DNA transcription; ii) TGIF competitively binds DNA-binding sites, such as CBP/p300 and p160/SRC with the growth factor-activated nuclear receptor, leading to the inhibition of transcription. It is suggested that during the process of growth and proliferation of endothelial cells, the balance between DNA transcription factors determines DNA transcription and plays a critical role in cell growth. The protein distribution revealed by SDS-PAGE and 2-D electrophoresis showed that following transfection with siRNA, the dots of total protein and basic protein were significantly reduced in the endothelial cells, which suggested high protein synthesis, and reflected the reduction in the reproductive ability of the endothelial cells. The expression of pyruvate kinase that mediates sugar decomposition decreased $(22,23)$, and promoted the reduction in the expression of PLTP (24) conveyed by lipids, which cannot provide the necessary materials and energy for cell metabolism. FR- $\beta$ (22) regulates folic acid metabolism, and negatively mediates cell proliferation. The upregulated expression of FR- $\beta$ may be involved in promoting the synthesis and metabolism of DNA and proteins, as well as cell proliferation.

Endothelial cells migrate following proliferation, and are rearranged under the attack of blood flow to form capillary vessel-like structures. In addition, it has been demonstrated that endothelial cells release bFGF during migration, which promotes the proliferation of endothelial cells. To further assess the pro-proliferation of enolase-1 in HUVECs, the mitosis and migration of HUVECs were observed using time-lapse video microscopy (22). Analysis of the extension of endothelial cell cytoplasm during mitosis showed that the number of endothelial cells transfected with siRNA during mitosis was greater than the non-transfected cells; however, the mobility was weaker. In addition, in vitro study of angiogenesis revealed that the endothelial cells extended slightly, which then formed small-volume tube-like structure with high density. Proteomics analysis revealed reduced $\mathrm{C} 1$ esterase $(25,26) . \mathrm{C} 1$ esterase, which was first identified in erythrocytes, facilitates the adhesion of endothelial cells to leucocytes and mediates the metabolism of the vascular matrix. The present study showed that $\mathrm{C} 1$ esterase promoted the adhesion between endothelial cells and the vascular matrix, the entension and adhesion to the vascular wall of the endothelial cell cytoplasm, and the adhesion and proliferation of the endothelial cells. In addition, reduced stomatin expression was observed. Stomatin is an integral membrane protein (26), which negatively mediates the membrane permeability of monovalent positive ions and is involved in the regulation of the assembly and organization of intracellular cytoskeletal proteins such as actin, and is closely associated with cell proliferation and migration.

Enolase-1 expression is closely related to tumor progression. In many tumors, the increased expression of enolase is a marker and treatment response marker. Jiang et al (27) demonstrated that IL-8, desmin and enolase-1 acted as the central elements in colon cancer susceptibility. Zhang et al (3) found that HER-2/neu signaling may result, directly or indirectly, in the enhanced activation of various metabolic, stressresponsive, antioxidative, and detoxification processes within the breast tumor microenvironment and hypothesized that the identified changes such as the increase in enolase expression were likely to drive cell proliferation and tissue invasion. In infiltrating ductal carcinomas of the breast, enolase-1 is overexpressed in tumors when compared with normal tissues (28). It has previously reported that enolase-1 and apolipoprotein-A may be useful as diagnostic markers of lung adenocarcinomas in clinical trials (29).

Currently, the non-surgical treatment of tumors mainly involves radiotherapy and chemotherapy, and radiotherapy and chemotherapy should be performed in the majority of surgeries for tumors. Insensitivity to radiotherapy and chemotherapy for tumors may emerge during hypoxia $(30,31)$. Oxygen plays a critical role in the production of free radicals following radiation. The oxygen-enriched cells are susceptible to radiotherapy, and are killed easily by radioactive rays, while hypoxic cells are tolerant to radiation, and are not killed. The issue of insufficient blood supply and a high percentage of hypoxic cells is often encountered in tumor tissue. Certain cancer cells evade radiation injury and cannot be effectively killed, which increases the risk of re-occurrence. MDA-MB-231 is a highly invasive breast cancer cell line.

The present study showed that the growth of tumor tissues and reduction in host body weight emerged rapidly after the injection of the cells to nude mice. However, in mice injected with the cancer cells transfected with enolase-1, a significant reduction in tumor growth, tumor size and weight was observed, and activity in the mice was not significantly affected. Following treatment with radioactive rays, tumor size was significantly reduced in both groups, although more notably in the transfected group. The efficacy was remarkable, although there was no significant difference observed compared with that prior to treatment. The data indicate that the inhibition of enolase-1 expression increases tolerance to hypoxia in tumor cells, which manifests slow cell growth, increased apoptosis and minor tumor size. Moreover, hypoxia is relatively weak 
within tissues, leading to fragile hypoxia-induced angiogenesis and low vascular density, and the efficacy of radiotherapy is also enhanced.

\section{Acknowledgements}

This study was financially supported by Grant no. Y2008C34 from the Natural Science Foundation of Shandong. We appreciate the valuable comments from other members of our laboratory.

\section{References}

1. Gordan JD and Simon MC: Hypoxia-inducible factors: central regulators of the tumor phenotype. Curr Opin Genet Dev 17: 71-77, 2007.

2. España L, Martín B, Aragüés R, et al: Bcl-x(L)-mediated changes in metabolic pathways of breast cancer cells: from survival in the blood stream to organ-specific metastasis. Am J Pathol 167: 1125-1137, 2005.

3. Zhang D, Tai LK, Wong LL, Chiu LL, Sethi SK and Koay ES: Proteomic study reveals that proteins involved in metabolic and detoxification pathways are highly expressed in HER-2/neupositive breast cancer. Mol Cell Proteomics 4: 1686-1696, 2005.

4. Wu F, Wang QM, Fan GC, Chen JZ and Chen HP: Proteomic analysis of paclitaxel-induced apoptosis in MCF-7 human breas carcinoma cells. Zhonghua Zhong Liu Za Zhi 28: 418-421, 2006 (In Chinese)

5. Dowling P, Meleady P, Dowd A, Henry M, Glynn S and Clynes M: Proteomic analysis of isolated membrane fractions from superinvasive cancer cells. Biochim Biophys Acta 1774: 93-101, 2007.

6. Yoo BS and Regnier FE: Proteomic analysis of carbonylated proteins in two-dimensional gel electrophoresis using avidinfluorescein affinity staining. Electrophoresis 25: 1334-1341, 2004.

7. Cavdar Z, Oktay G, Egrilmez MY, et al: In vitro reoxygenation following hypoxia increases MMP-2 and TIMP-2 secretion by human umbilical vein endothelial cells. Acta Biochim Pol 57: 69-73, 2010.

8. Scharte M, Han X, Bertges DJ, Fink MP and Delude RL: Cytokines induce HIF-1 DNA binding and the expression of HIF-1-dependent genes in cultured rat enterocytes. Am J Physiol Gastrointest Liver Physiol 284: G373-G384, 2003.

9. Somiari RI, Sullivan A, Russell S, et al: High-throughput proteomic analysis of human infiltrating ductal carcinoma of the breast. Proteomics 3: 1863-1873, 2003.

10. Choi HJ, Eun JS, Kim BG, Kim SY, Jeon H and Soh Y: Vitexin, an HIF-1alpha inhibitor, has anti-metastatic potential in PC12 cells. Mol Cells 22: 291-299, 2006.

11. Yu EZ, Li YY, Liu XH, et al: Antiapoptotic action of hypoxiainducible factor- $1 \alpha$ in human endothelial cells. Lab Invest 84 553-561, 2004.

12. Roland I, Minet E, Ernest I, et al: Identification of hypoxiaresponsive messengers expressed in human microvascular endothelial cells using differential display RT-PCR. Eur J Biochem 267: 3567-3574, 2000.

13. Li A, Li H, Jin G and Xiu R: A proteomic study on cell cycle progression of endothelium exposed to tumor conditioned medium and the possible role of cyclin D1/E. Clin Hemorheol Microcirc 29: 383-390, 2003.
14. Li A, Li H, Zhang J, Jin G and Xiu R: The mitogenic and antiapoptotic activity of tumor conditioned medium on endothelium. Clin Hemorheol Microcirc 29: 375-382, 2003.

15. Jaffe EA, Nachman RL, Becker CG and Minick CR: Culture of human endothelial cells derived from umbilical veins. Identification by morphologic and immunologic criteria. J Clin Invest 52: 2745-2756, 1973.

16. Hewett PW: Identification of tumour-induced changes in endothelial cell surface protein expression: an in vitro model. Int J Biochem Cell Biol 33: 325-335, 2001.

17. Jiang BH, Agani F, Passaniti A and Semenza GL: V-SRC induces expression of hypoxia-inducible factor 1 (HIF-1) and transcription of genes encoding vascular endothelial growth factor and enolase 1: involvement of HIF-1 in tumor progression. Cancer Res 57: 5328-5335, 1997.

18. Kim J and Dang CV: Multifaceted roles of glycolytic enzymes. Trends Biochem Sci 30: 142-150, 2005.

19. Kircher SG, Kim SH, Fountoulakis M and Lubec G: Reduced levels of DEAD-box proteins DBP-RB and p72 in fetal Down syndrome brains. Neurochem Res 27: 1141-1146, 2002.

20. Wotton D, Knoepfler PS, Laherty CD, Eisenman RN and Massagué J: The Smad transcriptional corepressor TGIF recruits mSin3. Cell Growth Differ 12: 457-463, 2001.

21. Melhuish TA, Gallo CM and Wotton D: TGIF2 interacts with histone deacetylase 1 and represses transcription. J Biol Chem 276: 32109-32114, 2001.

22. Nadimpalli R, Yalpani N, Johal GS and Simmons CR: Prohibitins, stomatins, and plant disease response genes compose a protein superfamily that controls cell proliferation, ion channel regulation, and death. J Biol Chem 275: 29579-29586, 2000.

23. Graven KK, Molvar C, Roncarati JS, Klahn BD, Lowrey S and Farber HW: Identification of protein disulfide isomerase as an endothelial hypoxic stress protein. Am J Physiol Lung Cell Mol Physiol 282: L996-L1003, 2002.

24. Nowak T and Suelter C: Pyruvate kinase: activation by and catalytic role of the monovalent and divalent cations. Mol Cell Biochem 35: 65-75, 1981.

25. Desrumaux C, Deckert V, Athias A, et al: Plasma phospholipid transfer protein prevents vascular endothelium dysfunction by delivering $\alpha$-tocopherol to endothelial cells. FASEB J 13: 883-892, 1999

26. Sun XL, Murphy BR, Li QJ, et al: Transduction of folate receptor cDNA into cervical carcinoma cells using recombinant adenoassociated virions delays cell proliferation in vitro and in vivo. $\mathrm{J}$ Clin Invest 96: 1535-1547, 1995.

27. Jiang W, Li X, Rao S, et al: Constructing disease-specific gene networks using pair-wise relevance metric: application to colon cancer identifies interleukin 8, desmin and enolase 1 as the central elements. BMC Syst Biol 2: 72, 2008.

28. Kabbage M, Chahed K, Hamrita B, et al: Protein alterations in infiltrating ductal carcinomas of the breast as detected by nonequilibrium $\mathrm{pH}$ gradient electrophoresis and mass spectrometry. J Biomed Biotechnol 2008: 564127, 2008.

29. Rütters H, Zürbig P, Halter R and Borlak J: Towards a lung adenocarcinoma proteome map: Studies with SP-C/c-raf transgenic mice. Proteomics 6: 3127-3137, 2006.

30. Sasabe E, Zhou X, Li D, Oku N, Yamamoto T and Osaki T: The involvement of hypoxia-inducible factor-1alpha in the susceptibility to gamma-rays and chemotherapeutic drugs of oral squamous cell carcinoma cells. Int J Cancer 120: 268-277, 2007.

31. Moulder JE and Rockwell S: Tumor hypoxia: its impact on cancer therapy. Cancer Metastasis Rev 5: 313-341, 1987. 